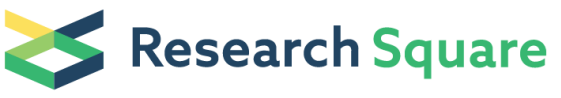 \\ Preprints are preliminary reports that have not undergone peer review. \\ They should not be considered conclusive, used to inform clinical practice, or referenced by the media as validated information.
}

\section{NLRP3 is Highly Expressed in Stomach Adenocarcinoma and May Serve as a Novel Prognostic Biomarker}

\author{
Chenxi Yuan \\ Qingdao University \\ Qingwei Wang \\ Qilu Hospital \\ Yipeng Song \\ Qingdao University \\ Jinming Yu ( $\nabla$ sdyujinming@163.com) \\ Shandong Cancer Hospital and Institute, Shandong First Medical University, Shandong Academy of Medical Sciences
}

\section{Research Article}

Keywords: Stomach adenocarcinoma, NLRP3, Biomarker,Tumor infiltrating lymphocytes, Bioinformatics analysis

Posted Date: December 29th, 2021

DOI: https://doi.org/10.21203/rs.3.rs-1199715/v1

License: (1) This work is licensed under a Creative Commons Attribution 4.0 International License. Read Full License 


\section{Abstract}

Background: Stomach adenocarcinoma (STAD) is a common cancer type around the world. The prognosis in advanced patients is poor. Since NLRP3 was not extensively studied in the field of tumor, so we aimed to identify the impact of NLRP3 on STAD by bioinformatics analyses and in vitro experiments.

Methods: TCGA , kaplan-Meier Plotter and TIMER database were utilized in this study. We compared the expression of NLRP3 in different cancers and evaluated its influence on survival of gastric carcinoma patients. The correlations between clinical information and NLRP3 expression were analyzed using logistic regression. Clinicopathologic characteristics associated with overall survival in were analyzed by Cox regression. In addition, we explored the correlation between NLRP3 and immune infiltrates. GSEA and co-expressed gene with NLRP3 were also done in this study. Finally, we verified the NLRP3 expression in paired gastric cancer tissues and adjacent normal tissues by western blot and q-PCR. We also constructed NLRP3 overexpression model in gastric cancer cell line and observed cell proliferation ability.

Results: NLRP3 expressed disparately in gastric tumor tissues and normal tissues. Cox regression analysis indicated that upregulated NLRP3 was an independent prognostic factor for bad prognosis in STAD. Logistic regression analysis showed increased NLRP3 expression was significantly correlated with unfavorable clinicopathologic parameters such as higher T stage, higher histologic grade and worse survival outcome. Specifically, a positive correlation between increased NLRP3 expression and immune infiltrating level of various immune cells was observed.

Conclusion: Together with all these findings, increased NLRP3 expression correlates with poor prognosis, unfavorable clinicopathologic parameters and increased proportion of immune cells in STAD. In vitro analysis revealed that cell proliferation ability was enhanced in gastric cancer cells trasnfected with NLRP3 overexpression. These conclusions indicate that NLRP3 has great potential to serve as a biomarker for evaluating prognosis in patients diagnosed with gastric carcinoma.

\section{Introduction}

Stomach cancer is the fourth leading cause of cancer-related deaths worldwide[1],representing a substantial global health burden. Stomach adenocarcinoma(STAD) accounts for the most common $(>90 \%)$ histological type [2]. Since STAD is often diagnosed at an advanced stage, the prognoses were quite poor even though progress has been made in treatment[3,4].Therefore, further researches are necessary for exploring novel therapeutic targets and potential molecular pathways for stomach pathogenesis and progression.

NLR family, pyrin domain containing 3 (NLRP3) is one of the most extensively investigated nucleotide-binding and oligomerization domain (NOD)-like receptors (NLRs). It has the ability to react to a wide range of extrinsic and intrinsic ligands such as pathogen associated molecular patterns (PAMPs) and/or damage associated molecular patterns (DAMPs), which can interact with pattern recognition receptors (PRRs) expressed on immune cells, then initiate a series of events leading to anti-tumor immunity. NLRP3 together with apoptosis-associated speck-like protein (ASC) and caspase-1 form as NLRP3 inflammasome, which is responsible for the maturation and secretion of proinflammatory cytokines[5,6].Therefore, NLRP3 is considered as a crucial innate immune component that orchestrate host immune homeostasis. Although there exist some researches investigated the role of NLRP3 in cancers, results are still quite preliminary and seem uncertain. For instance, Ershaid N illustrated that NLRP3 could facilitate tumor growth by recruiting myeloid-derived suppressor cells (MDSCs), and altering the tumor microenvironment toward an immunesuppressive milieu [7]. On the contrary, study of Dupaul-Chicoine showed that NLRP3 could suppress the metastasis of colorectal cancer (CRC) in the liver by promoting tumoricidal activity of natural killer (NK) cells [8].In light of the conflicting effect of NLRP3 in respect of tumors, we focused on STAD for further research. We aimed to unravel whether NLRP3 posed a positive or negative effect on tumor prognosis and tumor microenvironment (TME).

In this study, we comprehensively analyzed the expression of NLRP3 in tumor tissues and matched normal tissues, its correlation with prognosis and clinical parameters, as well as the status of different tumor-infiltrating immune cells in STAD. Our results confirmed the importance of NLRP3 in STAD prognosis and the close relationship between NLRP3 and human immune system. The workflow of our study is presented in Fig. 1.

Page 2/24 


\section{Materials And Methods}

\section{NLRP3 gene expression analysis}

To determine the difference of NLRP3 expression in several tumor and normal tissues, we obtained the mRNA levels (level 3) of NLRP3 from The Cancer Genome Atlas $\triangle T C G A \otimes()$ [9] and GTEx projects. The clinical information of STAD patients was also acquired from TCGA database. The threshold was determined as follows: fold change of 1.5, P-value of 0.001 , and gene ranking of all.

\section{Survival analysis}

To evaluate the prognostic value of NLRP3 in STAD patients, Kaplan-Meier Plotter ( ) was used through Hazard ratios (HRs) of 95\%, confidence intervals (Cls), and log-rank p-value.Kaplan-Meier Plotter is an online comprehensive database that contains gene expression and clinical data for 21 types of tumors[10] .

\section{Correlation analysis of NLRP3 and clinicopathological features}

We downloaded the clinicopathological data of patients with gastric carcinoma from TCGA database. The included patients were divided into high and low NLRP3 expression groups according to the median NLRP3 expression value. The collected clinical data involved age, sex, race, grade, TNM stage, lymph node metastasis, distant metastasis and vital status. The association between NLRP3 expression level and the clinicopathological parameters was analyzed using logistic regression.

\section{Evaluation of tumor-infiltrating immune cells}

For reliable immune score evaluation, we used an R software package named immuneeconv that integrated six latest algorithms, including TIMER, xCell, MCP-counter, CIBERSORT, EPIC and quanTIseq. We also evaluated the relationship between NLRP3 and crucial markers of several immune cells in TIMER database (https://cistrome.shinyapps. io/timer/) [11].The gene markers included markers of CD8+ T cells, T cells (general), B cells, neutrophils, natural killer (NK) cells, T regulatory (Treg)cells, T-helper 1 (Th1) cells, T-helper 2 (Th2) cells, follicular helper T (Tfh) cells, T-helper 17 (Th17) cells, exhausted T cells and Mast cells etc. The Spearman method was used to determine the correlation coefficient. NLRP3 was used for the X-axis, and other genes of interest are represented on the Y-axis.

\section{Gene set enrichment analysis}

Based on expression level of NLRP3, patients were divided into high and low groups. The pathways that were enriched by the top ranked genes in the two groups were detected by gene set enrichment analysis (GSEA). For each analysis, the number of gene set permutations was set to 1000. The nominal (NOM) P value, false discovery rate (FDR) and normalized enrichment score (NES) were used to identify the pathways enriched in each phenotype. Gene sets conforming to $\mid$ NES $\mid>1$ and NOM $p<0.05$ were deemed significant.

\section{Profiling of genes co-expressed with NLRP3}

For further study of the NLRP3-related molecular mechanisms, the co-expressed genes of NLRP3 were identified using DESeq2 in $R$ package to screen for proteins interacting with NLRP3. The top 6 significant genes correlated with NLRP3 in LUAD and SKCM patients were selected for further analysis, and their correlation with survival outcome was explored in the Gene Expression Profiling Interactive Analysis (GEPIA) (http:// gepia.cancer-pku.cn/index.html) [12].

\section{Statistical analysis}


SPSS 20.0 and R version 3.6.1 software were used for statistical analyses. The measurement data are presented as mean \pm SD. Paired sample t test were used to analyze the differential expression levels of NLRP3 mRNA between the tumor tissues and normal tissues from the TCGA database. The correlations between clinical characteristics and NLRP3 expression were analyzed using logistic regression. Univariate and multivariate analyses were performed using Cox proportional hazards regression model.The gene expression correlation was accessed by Spearman method. Statistically significant differences were considered when $\mathrm{P}<0.05$.

\section{Gastric Tissue Specimens}

In this study, 2 pairs of gastric cancer tissues and adjacent normal tissues were collected from the Yantai Yuhuangding hospital, Yantai, China. The collection and use of the samples were approved by the ethics committee of the Eighth Affiliated Hospital of Yantai Yuhuangding hospital.

\section{Cell Culture and NLRP3 Overexpression Cell Model Construction}

The gastric cancer cell lines SGC-7901 were obtained from Procell life science and Technology Co., Ltd (Wuhan, China). Cells were cultured in RPMI-1640 medium (Gibco, Los Angeles, CA, United States) with $10 \%$ fetal bovine serum (FBS, Gibco) and at $37^{\circ} \mathrm{C}$ in a 95\% air, $5 \% \mathrm{CO}_{2}$ humidified incubator. NLRP3 overexpression recombinant lentivirus (Genechem, Shanghai, China) were transfected into SGC-7901 cells according to manufacturer's instructions. Stable cell models were screened with $2.5 \mu \mathrm{g} / \mathrm{ml}$ puromycin.

\section{RT-PCR Analysis}

Human gastric cancer tissues and matched adjacent normal tissues were homogenized in Trizol (ThermoFisher Scientific) by tissue homogenizer (ThermoFisher Scientific). And the tissues and cells total RNA extracted by Trizol was used for reverse transcriptions via the primescript RT reagent (Takara Bio Inc., Japan) and then involved in the qPCR via SYBR Green PCR kit (Takara Bio Inc., Japan) and LightCycler96 Real-time PCR instrument (Roche). The analysis was conducted triply and GAPDH was used as internal reference. The following primers were used:NLRP3 forward primer: 5'-TCA CCA TGT GCT TCA TCC C-3';NRLP3 reverse primer: 5'-GGT GGT CTT GGA TGT CTG G-3';GAPDH forward primer: 5'-GAA CGA GCC GAG TGA AGC C-3'; GAPDH reverse primer: 5'-CTT TGA CTG CTT TCC CAC G-3'.

\section{Western blot}

Proteins of human gastric tissues were extracted using RIPA lysis buffer (Beyotime Institute of Biotechnology). Protein concentration in the cell lysate was determined using a bicinchoninic acid assay (Beyotime Institute of Biotechnology). A total of $30 \mu \mathrm{g}$ protein/lane were loaded and separated using 10\% SDS-PAGE, then transferred to polyvinylidene fluoride membranes (Merck KGaA), and blocked in PBS/Tween-20 containing 5\% bovine serum albumin (Millipore, Billerica, MA, USA) for $2 \mathrm{~h}$ at room temperature.

Subsequently, the membranes were incubated overnight at $4 \mathrm{C}$ with primary antibodies against the following: NLRP3 (cat. ab263899, Abcam), GAPDH (cat. Ab8245, Abcam). The membranes were then incubated with the goat anti-rabbit HRPconjugated secondary antibody (cat. Ab6721, Abcam) for $1 \mathrm{~h}$ at room temperature. To visualize protein bands, ECL system ( Millipore, Billerica, MA, USA) was used.

\section{Colony formation assay}

Gastric cells were seeded in 6-well plates at 1000 cells/well. Two weeks later, cell colonies were fixed with $4 \%$ paraformaldehyde for $30 \mathrm{~min}$ at room temperature and stained with $0.1 \%$ crystal violet for $30 \mathrm{~min}$ at room temperature. The numbers of colonies with $>50$ cells were counted under a light microscope. 


\section{Results}

\section{The mRNA Expression Levels of NLRP3 in Different Types of Human Cancers}

Initially, we evaluated NLRP3 expression levels in different human tumors and matched normal tissues by analyzing TCGA RNAseq data (Fig. 2A). As indicated in figure. 2A, NLRP3 was highly expressed in the tissues of bile duct cancer (CHOL), esophagus carcinoma (ESCA) and head and neck squamous cancer (HNSC) and significantly lower in bladder cancer (BLCA), thymoma (THYM) and lung squamous cell carcinoma (LUSC) tissues than in their respective normal tissues. These results demonstrated that NLRP3 was abnormally expressed in multiple tumors. In addition, we focused on the NLRP3 expression in stomach adenocarcinoma (STAD). We found that NLRP3 mRNA expression level was significantly higher in gastric adenocarcinoma tissue compared to normal counterparts (Fig. 2B).

\section{Prognostic Significance of NLRP3 in STAD}

Next, we investigated the prognostic value of NLRP3 expression in gastric adenocarcinoma using the Kaplan-Meier Plotter database. The results showed that overexpression of NLRP3 corresponded with unfavorable overall survival(OS) and progression free survival (PFS) in two different data sets of gastric cancer patients (Figure 3A-3B, OS HR 1.54, 95\% Cl 1.29-1.84, $p=1.1 \mathrm{e}-6$; HR 1.56, 95\% Cl 1.3-1.87, p=1.2e-6; Figure 3C-3D, PFS HR 1.59, 95\% Cl 1.28-1.98, p=2.7e-5;HR 1.63, 95\% Cl 1.27-2.09, p=0.00011). As we all know, recent advances in the field of gastric cancer have appeared a new classification based on molecular features (4), and this molecular is human epidermal growth factor receptor 2 (HER2). It has been demonstrated that HER2 is an important mediator of tumor cell proliferation and differentiation[13].Based on the status of HER2,one subtype is characterized by overexpression or amplification of HER2 and another is characterized by HER2 negative. As indicated in Figure.4, the high expression of NLRP3 predicted a worse prognosis no matter in HER2 negative or positive patients(Figure 4A-4B, Her-2 negative HR 1.47, 95\% Cl 1.17-1.85, p=0.00076;HR 1.62, 95\% Cl 1.28-2.05, p=4.7e-5; Figure 4C-4D, Her-2 positive HR 1.57, 95\% Cl 1.18-2.08, $\mathrm{p}=0.0016$; HR 1.37, 95\% Cl 1.06-1.78, p=0.016).Next, cox regression analysis was done to determine the factors that could influence the survival outcome of gastric patients. Univariate analysis indicated that T stage $(\mathrm{HR}=1.719,95 \% \mathrm{Cl} 1.131-2.612, \mathrm{P}-$ value $=0.011), \mathrm{N}$ stage $(\mathrm{HR}=1.925,95 \% \mathrm{Cl} 1.264-2.931$, $\mathrm{P}$-value $=0.002), \mathrm{M}$ stage $(\mathrm{HR}=2.254,95 \% \mathrm{Cl} 1.295-3.924 \mathrm{P}$-value $=0.004)$, pathological stage $(\mathrm{HR}=2.180,95 \% \mathrm{Cl} 1.388-3.423, \mathrm{P}$-value $<0.001)$, primary therapy outcome $(\mathrm{HR}=0.241,95 \% \mathrm{Cl} 0.165-0.352, \mathrm{P}$ value冈0.001), residual tumor $(\mathrm{HR}=3.445,95 \% \mathrm{Cl} 2.160-5.494, \mathrm{P}-\mathrm{value} \otimes 0.001)$ and the expression level of NLRP3 $(\mathrm{HR}=1.620,95 \%$ $\mathrm{Cl} 1.154-2.276$,P-value $=0.005$ ) were significantly associated with overall survival. Multivariate analysis showed that upregulated NLRP3 expression, stage IV, and progression of disease were independent prognostic factors of unfavorable prognosis(Table 1). For disease specific survival(DSS), univariate analysis revealed that T stage $(\mathrm{HR}=2.089,95 \% \mathrm{Cl} 1.192-3.660, \mathrm{P}-\mathrm{value}=0.011), \mathrm{N}$ stage $(H R=1.807,95 \% \mathrm{Cl} 1.075-3.0361 .264-2.931, \mathrm{P}$-value $=0.025)$, $\mathrm{M}$ stage $(H R=2.438,95 \% \mathrm{Cl} 1.221-4.870, \mathrm{P}$-value $=0.012)$, pathological stage $(\mathrm{HR}=2.320,95 \% \mathrm{Cl} 1.324-4.066, \mathrm{P}$-value $=0.003)$, primary therapy outcome $(\mathrm{HR}=0.136,95 \% \mathrm{Cl} 0.088-0.212, \mathrm{P}$ -

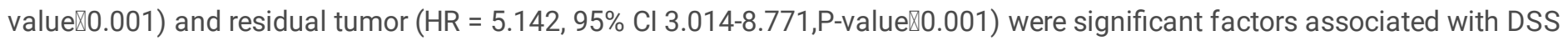
$\triangle$ Table2 $\nabla$.Multivariate analysis showed that primary therapy outcome and residue tumor were independent prognostic factors of DSS. In conclusion, we confirmed the prognostic value of NLRP3, its higher expression was associated with worse prognosis in gastric cancer patients.

\section{Association between NLRP3 expression and clinicopathologic variables}

Next, we evaluated the association between NLRP3 mRNA expression and several common clinicopathological parameters. Factors such as age, sex, T stage, lymph node metastasis, distant metastasis, pathologic stage, histologic grade and vital status were included for investigation. Based on the median value of NLRP3 expression level, patients were classified into high or low expression group. As summarized in Table 3, high expression of NLRP3 was closely associated with high T stage $(p=0.036)$, high histologic grade $(p<0.001)$ and worse survival outcome $(p=0.036)$ in patients with gastric carcinoma. No significant correlation between NLRP3 expression and other clinicopathological factors, such as age, gender, lymph node metastasis and distant metastasis was observed.The difference of NLRP3 expression level in different T stages, histologic grades and different survival 


\section{NLRP3-related signaling pathways in gastric carcinoma identified by GSEA}

In order to investigate the potential molecular function of NLRP3 in the carcinogenesis of gastric carcinoma, gene set enrichment analysis (GSEA) was conducted between samples with low and high NLRP3 expression to predict NLRP3-related signaling pathways. We focused on the top 6 significantly upregulated pathways (Figure. 6A-6F).

In STAD patients, the significantly upregulated pathways enriched in the high NLRP3 group included "Initial triggering of complement"pathway, "FCGR activation"pathway,"CD22 mediated BCR regulation"pathway,"Role of phosholipids in phagocytosis"pathway,"Immunoregulatory interactions between a Lymphoid and a non-Lymphoid cell”pathway, and"Antigen activates B Cell Receptor(BCR) leading to generation of second messengers" pathway.Interestingly , all these 6 pathways have close relationship with immune system, "Initial triggering of complement"pathway, "FCGR activation" pathway, "Role of phosholipids in phagocytosis" pathway belong to pathways of the innate immune system, and the following 3 pathways are related to adaptive immune system pathways. "Initial triggering of complement"pathway took part in the activation of complement, while "FCGR-mediated signaling in phagocytosis" pathway and "Role of phosholipids in phagocytosis" pathway acted in the process of phagocytosis. Three pathways closely connected to adaptive immune functions are crucial in modifying the response of lymphoid cells. For example,“CD22 mediated BCR regulation”pathway could regulate B lymphocyte function through both ligand-dependent and ligand-independent mechanisms[14].

\section{Analysis of genes co-expressed with NLRP3 in gastric carcinoma}

To further explore the possible effect of NLRP3 in gastric carcinoma, we tried to identify genes that positively co-expressed with NLRP3 with data downloaded from TCGA. The top 50 genes positively correlated with NLRP3 were selected and displayed as coexpression heatmap(Fig. 7). Additionally, we focused on the top 6 genes and verified their relationships with NLRP3 by scatter plots. As shown in Figure 8A-8F, expression of CASS4, PIK3R5, LCP2, LILRA1, MNDA and TM6SF1 were all positively correlated with the NLRP3 expression. investigated their relationship with overall survival outcome in LUAD or SKCM patients. Next,we tried to explore whether these co-expressed genes had an impact on overall survival(OS). Kaplan-Meier survival analysis showed that the expression of some co-expressed genes such as LILRA1, ITGAX, PIK3R5, RASGRP4, MYOIF and CSF1R was negatively correlated the overall survival (Figure. 9A-9F). Therefore, we believe that NLRP3 and its co-expressed genes collectively contribute to the worse survival in gastric carcinoma patients.

\section{Relationship between NLRP3 and tumor-infiltrating immune cells}

Previous analyses suggested tumor-infiltrating lymphocytes as independent predictors for survival in cancer patients[15]. Hence, we assessed the correlation between NLRP3 mRNA expression and immune infiltration status in gastric carcinoma.NK cells, macrophages, dendritic cells (DCs), B cells and T cells were included in the analysis. As indicated in Figure.10, NLRP3 expression level had significant positive correlations with infiltrating level of NK cells(Pख0.001), macrophages(Pख0.001), B cells(Pख0.001), T cells(Pख0.001), T helper cells(Pख0.001), total T helper cells(Pख0.001), T regulatory cells(Treg)(Pख0.001), $T$ follicular helper cells(Tfh) (P囚0.001),dendritic cells(DCs)(Pख0.001). However, the infiltrating level of T helper 1 cells (Th1), T helper 2 cells(Th2) and T helper 17 cells (Th17) had no relationship with the expression level of NLRP3. Further, we explored the correlations between NLRP3 and immune markers of a series of immune cells via TIMER databases. CD8+ T cells, T cells (general), B cells, neutrophils, dendritic cells, macrophages, monocyte and natural killer cells were included and different functional T cells, such as T helper 1(Th1) cells, T helper 2(Th2) cells, Tfh cells, T helper 17(Th17) cells, and Tregs, as well as exhausted T cells were analyzed. After the correlation adjustment by purity, the results revealed the NLRP3 expression level was significantly correlated with most immune marker sets of various immune cells and different T cells in gastric carcinoma. Markers of T cells, B cells, dendritic cells, macrophages as well as NK cells all showed significant correlations with NLRP3 expression (Table 4). We also found significant correlations between 
NLRP3 and marker genes of Treg and T cell exhaustion, such as PD-1, CTLA4, LAG3, and TIM3. In conclusion, these results demonstrated NLRP3 level was strongly correlated with immune infiltration, suggesting that NLRP3 may play a critical role in the gastric tumor microenvironment. Further studies need to be done to elucidate the exact role of NLRP3 in the carcinogenesis and tumor progression of gastric carcinoma.

\section{Verification of the Expression of NLRP3 in gastric cancer samples}

Based on the above bioinformatics analysis, we verified the expression of NLRP3 in two gastric cancer samples. The results of western blot showed that the relative expression level of NLRP3 was significantly higher in gastric cancer samples, compared with adjacent normal tissues (Figure 11A). Then the NLRP3 expression level was detected by qRT-PCR, and we found the relative mRNA level was higher in tumor tissues than in paired normal tissues (Figures 11B). The result demonstrated that NLRP3 might be identified as a biomarker for gastric cancer.

\section{NLRP3 Promotes Cell Proliferation in gastric cancer ells}

To characterize NLRP3 function involved in tumor progression, colony formation examination was performed.We used a common human gastric adenocarcinoma cell line SGC-7901 for in vitro study. Expression level of NLRP3 was upregulated by transfection of NLRP3-expressing lentivirus. As shown in Figure. 12, in NLRP3 overexpressed SGC-7901 cells, the number of successfully formed clones was significantly higher than vector-transfected cells, indicating that increased NLRP3 could significantly promote colony formation. The results suggested that NLRP3 promote cell proliferation in human gastric carcinoma cells.

\section{Discussion}

NLRP3 is the sensor component of the NLRP3 inflammasome, and it has the ability to response to pathogens and other damageassociated signals[16,17].Based on our study, we find there exist significant differences between tumor tissues and paired normal tissues in various cancers. Further investigations demonstrate that an up-regulated NLRP3 expression is associated with worse prognosis in gastric carcinoma. Moreover, the increased level of NLRP3 is correlated with unfavorable clinicopathological characteristics including higher tumor stage, higher histologic grade and worse survival outcome. It is revealed that diverse immune marker sets and immune infiltration status are correlated with NLRP3 expression in gastric carcinoma. In vitro experiments demonstrated that NLRP3 overexpression promoted the proliferation of gastric tumor cells. In conclusion, we hypothesize that NLRP3 has a potential influence on tumor immunology and serves as a promising prognostic biomarker for gastric carcinoma.

To understand the molecular functions and underlying mechanisms of NLRP3 better, GSEA was performed to investigate the pathways enriched in samples with high expression of NLRP3. We focused on the top 6 upregulated pathways. Interestingly, all these 6 pathways belonged to the category of "immune system pathway", 3 were among adaptive immune system pathways and the other 3 were innate immune system pathways. "Immunoregulatory interactions between a Lymphoid and a non-Lymphoid cell pathway" participates in adaptive immune response, the interactions between lymphoid and non-lymphoid cells are quite complicated, which require cooperation of a number of receptors and cell adhesion molecules [18,19]. The common receptor-ligand pairs include TCR-MHC1, CD40L-CD40, CD226-Nectin2, etc. "CD22 mediated BCR regulation pathway" also plays an important part in adaptive immune function. CD22 is a glycoprotein found on the surface of B cells and it is a member of the sialic acid-binding Ig-like lectin (Siglec) family [20].There is convincing evidence showing that CD22 can tightly regulate the activation of B cells[20,21]."Fc gamma receptor activation pathway" functions as an event of phagocytosis, which is a critical innate immune response eliminating the invaded infectious agents. Phagocytosis is an extremely complex process including phagocytic cup formation, particle engulfment, and the release of proinflammatory mediators such as cytokines and reactive oxygen species[22].In addition, another enriched pathway in STAD is "Antigen activates B Cell Receptor (BCR) leading to generation of second messengers", indicating that NLRP3 is involved in BCR signaling. In conclusion, the above results confirm that NLRP3 is closely correlated with immune functions. 
Next, we explored genes co-expressed with NLRP3 in STAD. The top 6 correlated genes were CASS4, PIK3R5, LCP2, LILRA1,MNDA and TM6SF1. CASS4 is a member of CAS family, previous studies reported the important roles for the CAS (BCAR1, NEDD9, EFS,and CASS4) proteins in tumorigenesis and other pathological states.It has been demonstrated that CASS4 overexpression correlated with positive lymph node metastasis and poor prognosis in non-small cell lung cancer[23]. PIK3R5 is a member of $\mathrm{PI} 3 \mathrm{Ks}$, it plays critical roles in cell growth. The expression of PIK3R5 was significantly increased in acute myeloid leukemia (AML) tissue samples compared with healthy controls. Moreover,PIK3R5 could facilitate AML cells viability and inhibit apoptosis by regulating the function of HCP5[24]. We believe these co-expressed genes contributed to the worse prognosis caused by high expression of NLRP3 in gastric adenocarcinoma.

We also investigated the impact of NLRP3 expression on the status of tumor infiltrating lymphocytes in STAD. Significantly positive correlation was found between NLRP3 expression and high infiltration of various immune cells, immunocytes considered as indispensable effectors for anti-tumor responses and immune-suppressive cells inducing immunological tolerance are both involved. NK cells, CD8+ T cells are typical cells closely associated with effective cancer treatment [25] and their high infiltration is considered to be associated with a better survival outcome in cancer patients. In gastric cancer patients, we observed that high NLRP3 level is concomitantly correlated with high infiltration of immunocytes with inhibitory effects such as T regulatory cells and tumor associated macrophages [26].Therefore, we inferred the accumulation of these immune suppressive lymphocytes partially led to the worse outcomes of gastric cancer patients with high NLRP3.

In summary, our study confirmed that increased NLRP3 expression correlated with worse prognosis in gastric carcinoma patients. Meanwhile, NLRP3 expression levels were closely associated with the level of tumor-infiltrating immune cells and several unfavorable clinicopathologic parameters such as high tumor grade. Therefore, NLRP3 had an essential influence on immune infiltration and had the potential to become a novel predictor to evaluate prognosis and immune infiltration for STAD patients. Our in vitro experiment proved that overexpression of NLRP3 could promote the proliferation ability of SGC-7901 gastric cancer cells. We think more extensive researches need to be done to elucidate the possible mechanisms for NLRP3 in influencing tumor microenvironment and tumor-immune interactions in gastric carcinoma.

\section{Conclusion}

In this study, we observed NLRP3 mRNA was differentially expressed in tumor tissues and normal tissues in several cancers. High NLRP3 expression was associated with shorter overall survival in stomach adenocarcinoma. NLRP3 may have great potential to serve as a biologic predictor of prognosis for gastric cancer patients.

\section{Declarations}

\section{Acknowledgements}

Not applicable.

\section{Authors' contributions}

Conception and design of the work, Chenxi Yuan and Yipeng Song; acquisition and analysis of data, Chenxi Yuan ; interpretation of data, Chenxi Yuan and Qingwei Wang; writing and preparing the original draft, Chenxi Yuan; supervision, Jinming Yu and Yipeng Song; funding acquisition, Jinming Yu. All authors have read and agreed to the published version of the manuscript and to have agreed to both be personally accountable for the author's contributions and ensure to answer any questions related to the accuracy or integrity of any part of the work. All authors read and approved the final manuscript.

\section{Funding}

This work was funded by the grants from National Key Research and Development Projects of China (2018YFC1312201). 


\section{Availability of data materials}

The original contributions presented in the study are included in the article, further inquiries can be directed to the corresponding authors.

\section{Ethics approval and consent to participate}

This study was ratified by the Ethics Committee of Yantai Yuhuangding hospital. And this study does not involve any animal experiments.

\section{Consent for publication}

Not applicable.

\section{Competing interests}

The authors declare that they have no competing interests

\section{References}

1. Sung H, Ferlay, J, Siegel, RL, et al. Global cancer statistics 2020: GLOBOCAN estimates of incidence and mortality worldwide for 36 cancers in 185 countries. CA Cancer J Clin 2021; 71: 209-49.

2. Ajani JA, Lee J, Sano T, Janjigian YY, Fan D, Song S. Gastric adenocarcinoma. Nat Rev Dis Primers 2017; 3: 17036.

3. Jones JO, Smyth EC. Gastroesophageal cancer: Navigating the immune and genetic terrain to improve clinical outcomes. Cancer Treat Rev 2020; 84: 101950.

4. Joshi SS, Badgwell BD. Current treatment and recent progress in gastric cancer. CA Cancer J Clin. 2021;71:264-279.

5. Abderrazak A, Syrovets T, Couchie D, El Hadri K, Friguet B, Simmet T, et al. NLRP3 inflammasome: from a danger signal sensor to a regulatory node of oxidative stress and inflammatory diseases. Redox Biol. 2015;4:296-307

6. Alexandre YO, Cocita CD, Ghilas S, Dalod M. Deciphering the role of DC subsets in MCMV infection to better understand immune protection against viral infections. Front Microbiol. 2014; 5:378.

7. Ershaid, N. et al. NLRP3 inflammasome in fibroblasts links tissue damage with inflammation in breast cancer progression and metastasis. Nat. Commun.10,4375 (2019).

8. Dupaul-Chicoine, J. et al. The Nlrp3 inflammasome suppresses colorectal cancer metastatic growth in the liver by promoting natural killer cell tumoricidal activity. Immunity43, 751-763 (2015).

9. Cancer Genome Atlas Network (2012). Comprehensive molecular portraits of human breast tumours. Nature 490, 61-70.

10. Szász AM, Lánczky A, Nagy Á, Förster S, Hark K, Green JE, Boussioutas A, Busuttil R, Szabó A, Győrffy B: Cross-validation of survival associated biomarkers in gastric cancer using transcriptomic data of 1,065 patients, Oncotarget, DOI: 10.18632/oncotarget.10337.

11. Li T, Fan J, Wang B, Traugh N, Chen Q, Liu JS, Li B, Liu XS. TIMER: A Web Server for Comprehensive Analysis of TumorInfiltrating Immune Cells. Cancer Res. 2017; 77:e108-10.

12. Tang Zefang,Li Chenwei,Kang Boxi et al. GEPIA: a web server for cancer and normal gene expression profiling and interactive analyses.[J] .Nucleic Acids Res, 2017, 45: W98-W102.

13. Yang YM, Hong P, Xu WW, He QY, Li B. Advances in targeted therapy for esophageal cancer. Signal Transduct Target Ther. 2020;5:229.

14. Poe Jonathan C, Fujimoto Yoko,Hasegawa Minoru et al. CD22 regulates B lymphocyte function in vivo through both liganddependent and ligand-independent mechanisms.[J] .Nat Immunol, 2004, 5: 1078-87. 
15. Ohtani H. Focus on TILs: prognostic significance of tumor infiltrating lymphocytes in human colorectal cancer. Cancer Immun. (2007) 7:4.

16. Swanson Karen V, Deng Meng, Ting Jenny P-Y, The NLRP3 inflammasome: molecular activation and regulation to therapeutics. [J]. Nat Rev Immunol, 2019, 19: 477-489.

17. Ratajczak Mariusz Z, Kucia Magdalena, The Nlrp3 inflammasome - the evolving story of its positive and negative effects on hematopoiesis. [J]. Curr Opin Hematol, 2021, 28: 251-261.

18. Vivier Eric, Tomasello Elena, Baratin Myriam et al. Functions of natural killer cells. [J]. Nat Immunol, 2008, 9: 503-10.

19. Nedvetzki Shlomo, Sowinski Stefanie, Eagle Robert A et al. Reciprocal regulation of human natural killer cells and macrophages associated with distinct immune synapses. [J]. Blood, 2007, 109: 3776-85.

20. Jellusova Julia, Nitschke Lars, Regulation of B cell functions by the sialic acid-binding receptors siglec-G and CD22. [J]. Front Immunol, 2011, 2: 96.

21. Poe Jonathan C, Fujimoto Yoko, Hasegawa Minoru et al. CD22 regulates B lymphocyte function in vivo through both liganddependent and ligand-independent mechanisms. [J]. Nat Immunol, 2004, 5: 1078-87.

22. Joshi Trupti, Butchar Jonathan P, Tridandapani Susheela, Fc gamma receptor signaling in phagocytes. [J]. Int J Hematol, 2006, 84: 210-6.

23. Miao Yuan,Wang Liang,Liu Yang et al. Overexpression and cytoplasmic accumulation of Hepl is associated with clinicopathological parameters and poor prognosis in non-small cell lung cancer.[J].Tumour Biol, 2013, 34: 107-14.

24. Liu Yan,Jing Xue-Bing,Wang Zhen-Cheng et al. HCP5, as the sponge of miR-1291, facilitates AML cell proliferation and restrains apoptosis via increasing PIK3R5 expression.[J] .Hum Genomics, 2021, 15: 38.

25. Tang Yuting, Xu Qian, Hu Liang et al. Tumor Microenvironment-Derived R-spondins Enhance Anti-Tumor Immunity to Suppress Tumor Growth and Sensitize for Immune Checkpoint Blockade Therapy. [J]. Cancer Discov, 2021, undefined: undefined.

26. Marangoni Francesco, Zhakyp Ademi, Corsini Michela et al. Expansion of tumor-associated Treg cells upon disruption of a CTLA-4-dependent feedback loop. [J]. Cell, 2021, 184: 3998-4015.e19.

\section{Tables}

Table 1

Univariate and multivariate Cox regression analysis for overall survival(OS) in gastric carcinoma patients. 


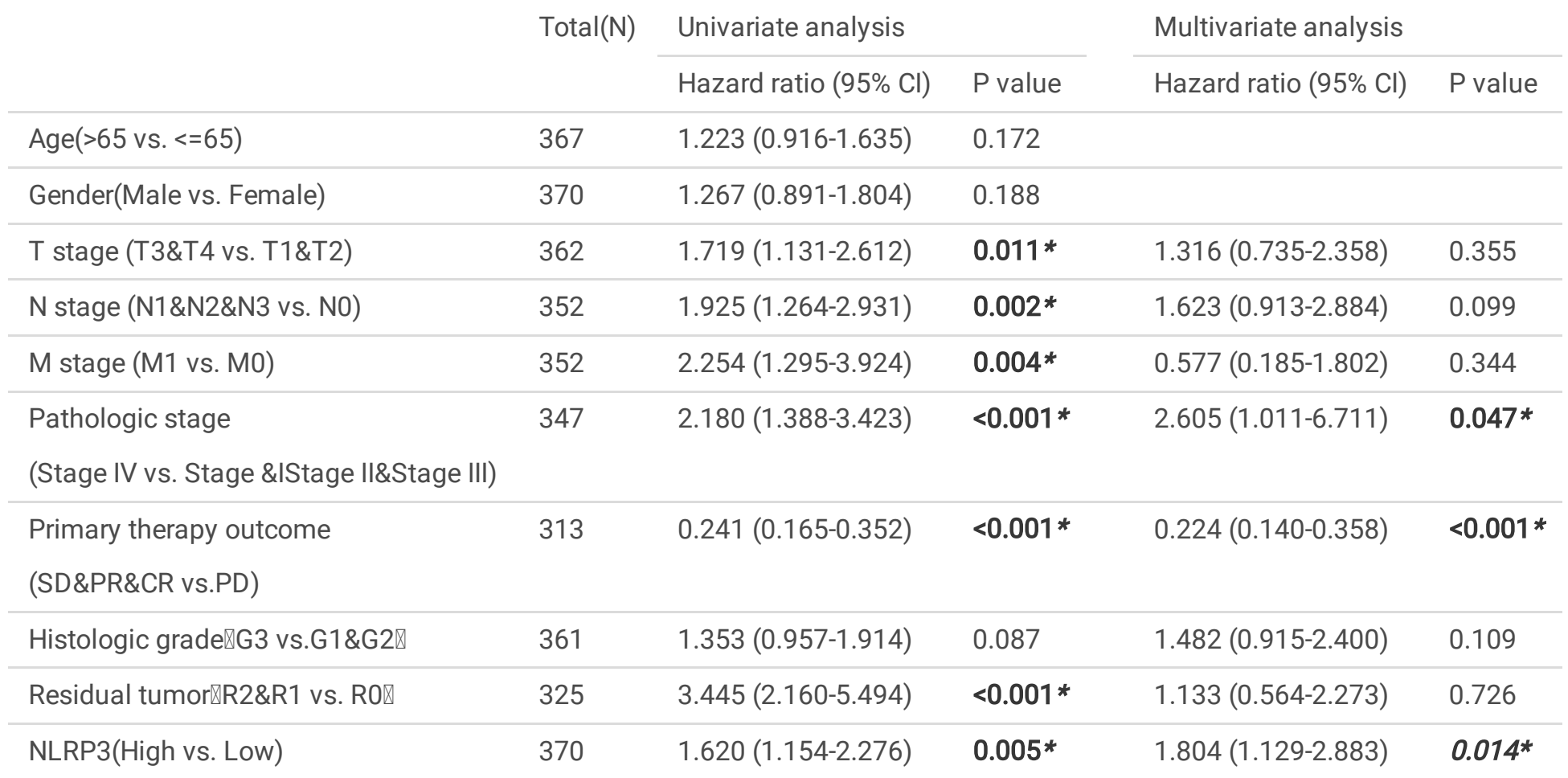

Table 2.

Univariate and multivariate Cox regression analysis for disease specific survival(DSS) in gastric cancer patients 


\begin{tabular}{|c|c|c|c|c|c|}
\hline \multirow[t]{2}{*}{ Characteristics } & \multirow[t]{2}{*}{ Total(N) } & \multicolumn{2}{|l|}{ Univariate analysis } & \multicolumn{2}{|l|}{ Multivariate analysis } \\
\hline & & Hazard ratio $(95 \% \mathrm{Cl})$ & $P$ value & Hazard ratio $(95 \% \mathrm{Cl})$ & $P$ value \\
\hline Age(>65 vs. <=65) & & $1.211(0.797-1.840)$ & 0.371 & & \\
\hline & 346 & & & & \\
\hline T stage(T3\&T4 vs. T1\&T2) & 345 & $2.089(1.192-3.660)$ & 0.010 & $1.405(0.731-2.698)$ & 0.308 \\
\hline N stage 『N1\&N2\&N3 vs. N0) & 334 & $1.807(1.075-3.036)$ & 0.025 & $1.295(0.663-2.528)$ & 0.449 \\
\hline M stage(M1 vs. M0) & 333 & $2.438(1.221-4.870)$ & 0.012 & $1.025(0.325-3.235)$ & 0.966 \\
\hline HER2囚High vs. Low $\rrbracket$ & 349 & $1.103(0.727-1.673)$ & 0.645 & & \\
\hline NLRP3(High vs. Low) & 349 & $1.163(0.764-1.770)$ & 0.482 & & \\
\hline $\begin{array}{l}\text { Pathologic stage } \\
\text { (Stage IV vs. Stage I\&Stage II\&Stage III) }\end{array}$ & 331 & $2.320(1.324-4.066)$ & 0.003 & $1.054(0.407-2.734)$ & 0.913 \\
\hline $\begin{array}{l}\text { Primary therapy outcome } \\
\text { (SD\&PR\&CR vs.PD) }\end{array}$ & 310 & $0.136(0.088-0.212)$ & $<0.001$ & $0.147(0.088-0.245)$ & $<0.001$ \\
\hline $\begin{array}{l}\text { Residual tumor } \\
\text { (R2\&R1 vs. R0囚 }\end{array}$ & 314 & $5.142(3.014-8.771)$ & $<0.001$ & $2.484(1.217-5.070)$ & 0.012 \\
\hline Histologic grade $\mathbb{G} 3$ vs.G1\&G2】 & 340 & $1.338(0.862-2.078)$ & 0.194 & & \\
\hline
\end{tabular}

Table 3

Association between NLRP3 expression status and several clinicopathologic variables. 


\begin{tabular}{|c|c|c|c|}
\hline Characteristic & Low expression of NLRP3 & High expression of NLRP3 & $\mathrm{p}$ \\
\hline$n$ & 187 & 188 & \\
\hline Age, n (\%) & & & 0.508 \\
\hline$<=65$ & $79(21.3 \%)$ & $85(22.9 \%)$ & \\
\hline$>65$ & $108(29.1 \%)$ & $99(26.7 \%)$ & \\
\hline Gender, n (\%) & & & 0.884 \\
\hline Female & $68(18.1 \%)$ & $66(17.6 \%)$ & \\
\hline Male & 119 (31.7\%) & $122(32.5 \%)$ & \\
\hline T stage, n (\%) & & & $0.036 *$ \\
\hline T1 & $15(4.1 \%)$ & $4(1.1 \%)$ & \\
\hline T2 & $36(9.8 \%)$ & $44(12 \%)$ & \\
\hline T3 & $90(24.5 \%)$ & $78(21.3 \%)$ & \\
\hline T4 & $46(12.5 \%)$ & $54(14.7 \%)$ & \\
\hline N stage, n (\%) & & & 0.449 \\
\hline NO & $61(17.1 \%)$ & $50(14 \%)$ & \\
\hline N1 & $50(14 \%)$ & $47(13.2 \%)$ & \\
\hline N2 & $36(10.1 \%)$ & $39(10.9 \%)$ & \\
\hline N3 & $32(9 \%)$ & $42(11.8 \%)$ & \\
\hline M stage, n (\%) & & & 1.000 \\
\hline MO & $164(46.2 \%)$ & $166(46.8 \%)$ & \\
\hline M1 & $12(3.4 \%)$ & $13(3.7 \%)$ & \\
\hline Pathologic stage, n (\%) & & & 0.618 \\
\hline Stage I & $31(8.8 \%)$ & $22(6.2 \%)$ & \\
\hline Stage II & $58(16.5 \%)$ & $53(15.1 \%)$ & \\
\hline Stage III & $73(20.7 \%)$ & $77(21.9 \%)$ & \\
\hline Stage IV & $18(5.1 \%)$ & $20(5.7 \%)$ & \\
\hline Primary therapy outcome, $\mathrm{n}(\%)$ & & & 0.678 \\
\hline PD & $37(11.7 \%)$ & $28(8.8 \%)$ & \\
\hline SD & $9(2.8 \%)$ & $8(2.5 \%)$ & \\
\hline PR & $1(0.3 \%)$ & $3(0.9 \%)$ & \\
\hline CR & $122(38.5 \%)$ & $109(34.4 \%)$ & \\
\hline Histologic grade, n (\%) & & & $<0.001 *$ \\
\hline G1 & $7(1.9 \%)$ & $3(0.8 \%)$ & \\
\hline G2 & 89 (24.3\%) & $48(13.1 \%)$ & \\
\hline G3 & $85(23.2 \%)$ & $134(36.6 \%)$ & \\
\hline OS event, n (\%) & & & $0.013^{*}$ \\
\hline
\end{tabular}




$\begin{array}{lll}\text { Alive } & 126(33.6 \%) & 102(27.2 \%) \\ \text { Dead } & 61(16.3 \%) & 86(22.9 \%)\end{array}$

Table 4

Correlation analysis between NLRP3 expression and gene markers of lymphocytes 


\begin{tabular}{|c|c|c|c|c|c|}
\hline \multirow[t]{3}{*}{ Description } & \multirow[t]{3}{*}{ Gene markers } & \multicolumn{4}{|l|}{ STAD } \\
\hline & & \multicolumn{2}{|l|}{ None } & \multicolumn{2}{|l|}{ Purity } \\
\hline & & Cor & $\mathbf{P}$ & Cor & $\mathbf{P}$ \\
\hline \multirow[t]{3}{*}{ Tell(general) } & CD3D & 0.512 & $3.61 e-29$ & 0.481 & $2.18 \mathrm{e}-23$ \\
\hline & CD3E & 0.524 & $1.27 e-30$ & 0.496 & $6.24 \mathrm{e}-25$ \\
\hline & CD2 & 0.597 & $2.15 e-41$ & 0.583 & $6.40 e-36$ \\
\hline \multirow[t]{2}{*}{ CD8+ T cell } & CD8A & 0.513 & $2.74 \mathrm{e}-29$ & 0.495 & $7.68 \mathrm{e}-25$ \\
\hline & CD8B & 0.343 & $7.26 \mathrm{e}-13$ & 0.324 & $9.74 \mathrm{e}-11$ \\
\hline \multirow[t]{5}{*}{ Th1 } & T-bet & 0.558 & $2.34 \mathrm{e}-35$ & 0.538 & $7.04 \mathrm{e}-30$ \\
\hline & STAT1 & 0.355 & $9.19 \mathrm{e}-14$ & 0.363 & $2.93 e-13$ \\
\hline & STAT4 & 0.664 & $4.67 e-54$ & 0.66 & $9.38 \mathrm{e}-49$ \\
\hline & IFN-y & 0.336 & $2.04 \mathrm{e}-12$ & 0.325 & $8.50 \mathrm{e}-11$ \\
\hline & TNF-a & 0.399 & $2.8 \mathrm{e}-17$ & 0.351 & $1.87 e-12$ \\
\hline \multirow[t]{4}{*}{ Th2 } & GATA3 & 0.414 & $1.27 e-18$ & 0.402 & $3.73 e-16$ \\
\hline & STAT6 & 0.28 & $6.18 \mathrm{e}-09$ & 0.3 & $2.64 \mathrm{e}-09$ \\
\hline & STAT5A & 0.571 & $3.3 e-37$ & 0.571 & $3.23 e-34$ \\
\hline & IL13 & 0.157 & $1.37 e-03$ & 0.162 & $1.58 \mathrm{e}-03$ \\
\hline \multirow[t]{2}{*}{ Th17 } & STAT3 & 0.549 & $5.2 \mathrm{e}-34$ & 0.554 & $6.68 e-32$ \\
\hline & IL17A & 0.031 & $5.35 e-01$ & 0.007 & $8.84 \mathrm{e}-01$ \\
\hline \multirow[t]{4}{*}{ Treg } & FOXP3 & 0.575 & $8.1 \mathrm{e}-38$ & 0.558 & $1.88 \mathrm{e}-32$ \\
\hline & CCR8 & 0.683 & $2.58 e-58$ & 0.683 & $1.94 \mathrm{e}-53$ \\
\hline & STAT5B & 0.552 & $1.87 e-34$ & 0.568 & $8.17 e-34$ \\
\hline & TGF $\beta$ & 0.516 & $1.49 \mathrm{e}-29$ & 0.505 & $5.70 \mathrm{e}-26$ \\
\hline \multirow[t]{4}{*}{ T cell exhaustion } & PD-1 & 0.459 & $5.13 e-23$ & 0.444 & $9.89 \mathrm{e}-20$ \\
\hline & CTLA4 & 0.497 & $3.03 e-27$ & 0.471 & $2.44 \mathrm{e}-22$ \\
\hline & TIM-3 & 0.747 & $3.33 e-75$ & 0.746 & $1.49 \mathrm{e}-68$ \\
\hline & LAG3 & 0.44 & $4.36 e-21$ & 0.424 & $5.98 \mathrm{e}-18$ \\
\hline \multirow[t]{2}{*}{ B cell } & CD19 & 0.385 & $4.44 \mathrm{e}-16$ & 0.35 & $2.43 e-12$ \\
\hline & CD79A & 0.413 & $1.57 e-18$ & 0.376 & $3.84 \mathrm{e}-14$ \\
\hline \multirow[t]{7}{*}{ Natural killer cell } & KIR2DL1 & 0.334 & $2.79 e-12$ & 0.339 & $1.27 \mathrm{e}-11$ \\
\hline & KIR2DL3 & 0.287 & $2.61 \mathrm{e}-09$ & 0.278 & $3.61 e-08$ \\
\hline & KIR2DL4 & 0.215 & $2.48 e-05$ & 0.257 & $1.09 \mathrm{e}-07$ \\
\hline & KIR3DL1 & 0.305 & $2.27 e-10$ & 0.298 & $3.13 e-09$ \\
\hline & KIR3DL2 & 0.325 & $1.24 \mathrm{e}-11$ & 0.311 & $6.02 \mathrm{e}-10$ \\
\hline & KIR3DL3 & 0.117 & $1.75 e-02$ & 0.137 & $7.56 \mathrm{e}-03$ \\
\hline & KIR2DS4 & 0.24 & $7.25 \mathrm{e}-07$ & 0.25 & $8.50 \mathrm{e}-07$ \\
\hline
\end{tabular}




\begin{tabular}{llllll} 
Dendritic cell & HLA-DPB1 & 0.578 & $2.07 \mathrm{e}-38$ & 0.556 & $4.03 \mathrm{e}-32$ \\
\hline & HLA-DQB1 & 0.418 & $5.77 \mathrm{e}-19$ & 0.379 & $2.30 \mathrm{e}-14$ \\
\hline & HLA-DRA & 0.573 & $1.47 \mathrm{e}-37$ & 0.559 & $1.78 \mathrm{e}-32$ \\
\hline & HLA-DPA1 & 0.569 & $5.12 \mathrm{e}-37$ & 0.553 & $9.49 \mathrm{e}-32$ \\
\hline & BDCA-1 & 0.54 & $1.02 \mathrm{e}-32$ & 0.504 & $8.43 \mathrm{e}-26$ \\
\hline & BDCA-4 & 0.7 & $2.22 \mathrm{e}-62$ & 0.69 & $9.06 \mathrm{e}-55$ \\
\hline Neutrophils & CD11C & 0.81 & $1.22 \mathrm{e}-97$ & 0.802 & $1.61 \mathrm{e}-86$ \\
\hline & CD66b & 0.127 & $9.46 \mathrm{e}-03$ & 0.14 & $6.42 \mathrm{e}-03$ \\
\hline & CD11b & 0.736 & $5.46 \mathrm{e}-72$ & 0.729 & $6.02 \mathrm{e}-64$ \\
\hline Monocyte & CCR7 & 0.627 & $8.42 \mathrm{e}-47$ & 0.608 & $1.03 \mathrm{e}-39$ \\
\hline & CD86 & 0.777 & $6.59 \mathrm{e}-85$ & 0.774 & $8.40 \mathrm{e}-77$ \\
\hline M1 Macrophage & iNOS & 0.177 & $2.95 \mathrm{e}-04$ & 0.159 & $1.85 \mathrm{e}-03$ \\
\hline & IRF5 & 0.416 & $8.27 \mathrm{e}-19$ & 0.421 & $1.13 \mathrm{e}-17$ \\
\hline TAM & COX2 & 0.286 & $2.79 \mathrm{e}-09$ & 0.261 & $2.67 \mathrm{e}-07$ \\
\hline M2 Macrophage & CD163 & 0.812 & $8.35 \mathrm{e}-99$ & 0.813 & $9.57 \mathrm{e}-91$ \\
\hline & VSIG4 & 0.697 & $1.16 \mathrm{e}-61$ & 0.701 & $2.71 \mathrm{e}-57$ \\
\hline & MS4A4A & 0.749 & $1.07 \mathrm{e}-75$ & 0.747 & $7.11 \mathrm{e}-69$ \\
\hline & CCL2 & 0.549 & $5.41 \mathrm{e}-34$ & 0.519 & $1.62 \mathrm{e}-27$ \\
\hline & IL10 & 0.581 & $7.39 \mathrm{e}-39$ & 0.573 & $1.93 \mathrm{e}-34$ \\
\hline & & 0.693 & $1.22 \mathrm{e}-60$ & 0.666 & $5.69 \mathrm{e}-50$
\end{tabular}

\section{Figures}

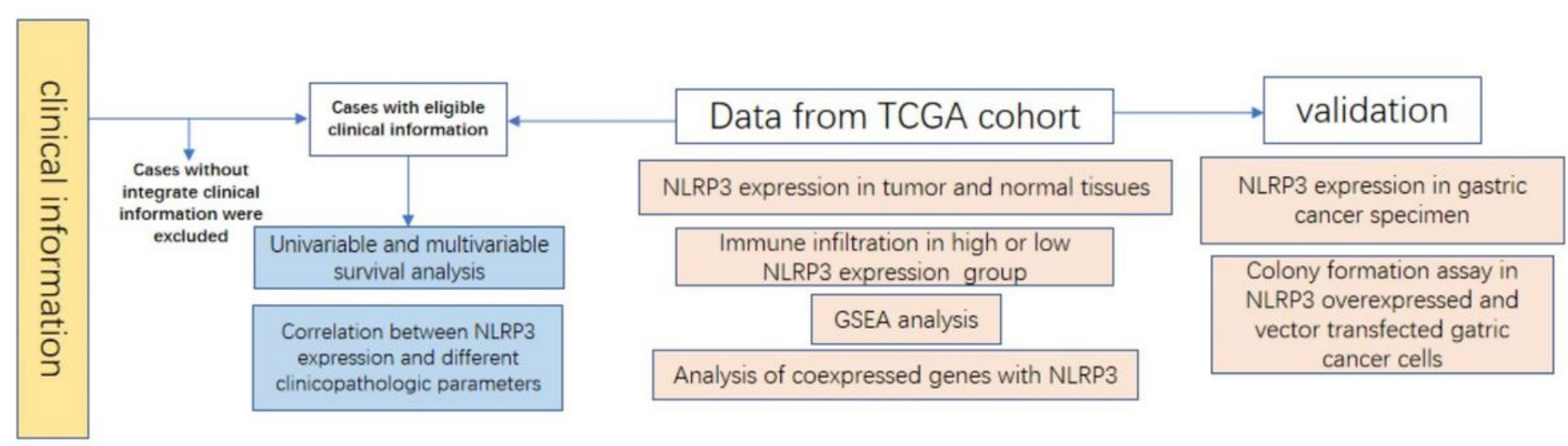

Figure 1

Workflow of this study. 


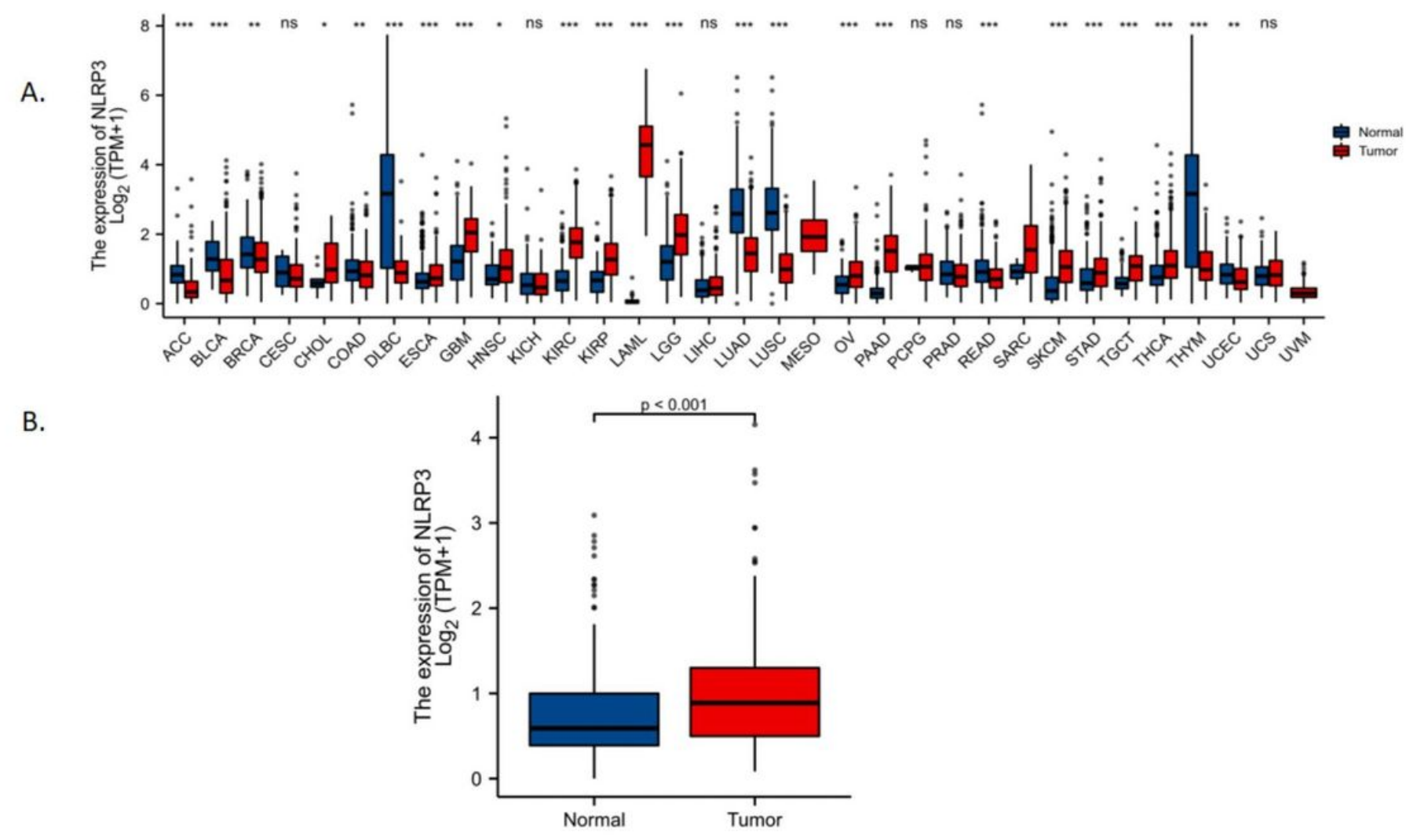

Figure 2

The expression level of NLRP3 in different types of human cancers. (A) Increased or decreased NLRP3 in data sets of different cancers compared with normal tissues. (B) NLRP3 mRNA levels in STAD tissues and normal stomach tissues in TCGA. ns, $p \geq 0.05$; $*, p<0.05 ; * *, p<0.01 ; * * *, p<0.001$. 

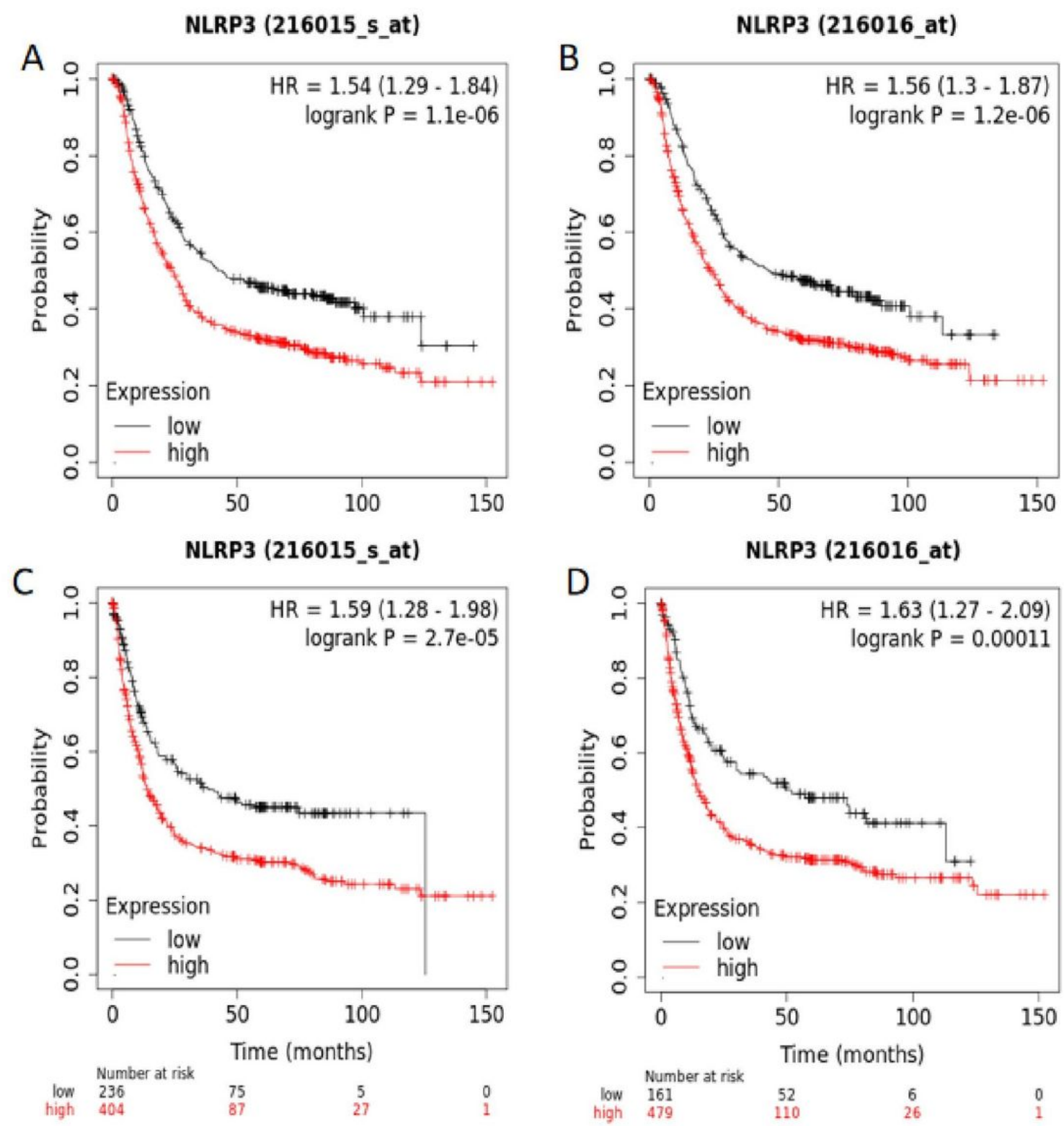

Figure 3

Kaplan-Meier survival curve analysis of the prognostic significance of high and low expression of NLRP3 in STAD. High expression was correlated with worse overall survival(OS)(A,B) and progression free survival(C,D). 

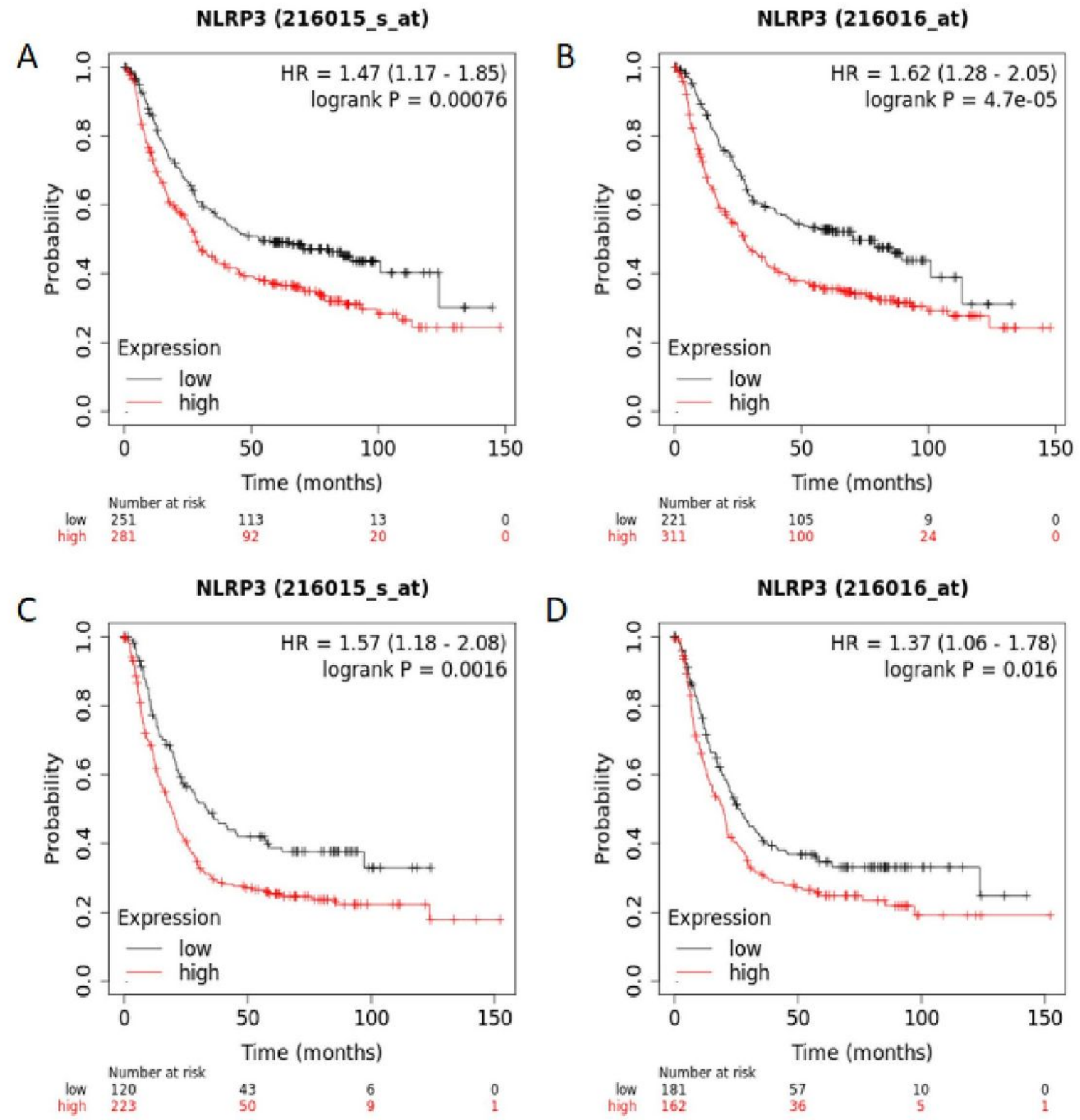

Figure 4

Overexpression of NLRP3 was associated with worse overall survival both in HER2 negative $(A, B)$ and positive $(C, D)$ gastric adenocarcinoma patients. 

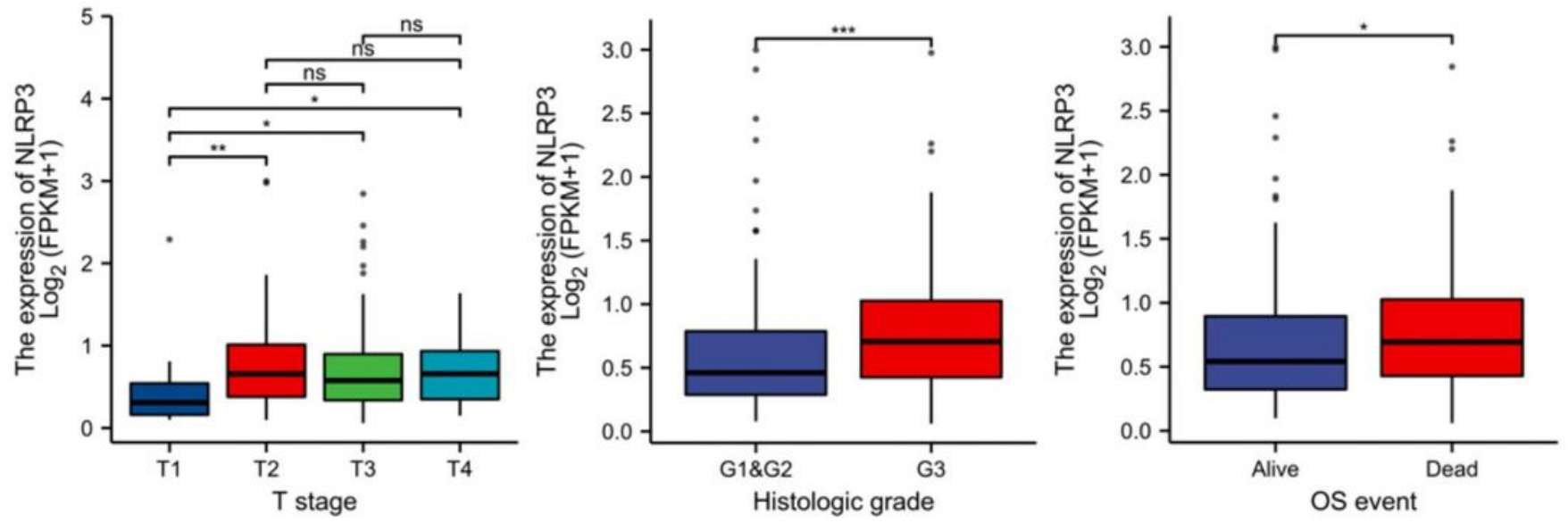

\section{Figure 5}

Correlationship between T stage(A), histologic grade(B) and survival event(C) and NLRP3 expression level in gastric cancer.Patients with high T stage, high histologic stage or worse survival outcome had high expression level of NLRP3; ${ }^{*} p<0.05$; $\star *, p<0.01 ; * \star \star, p<0.001, n s$, not significant, $p \geq 0.05$.
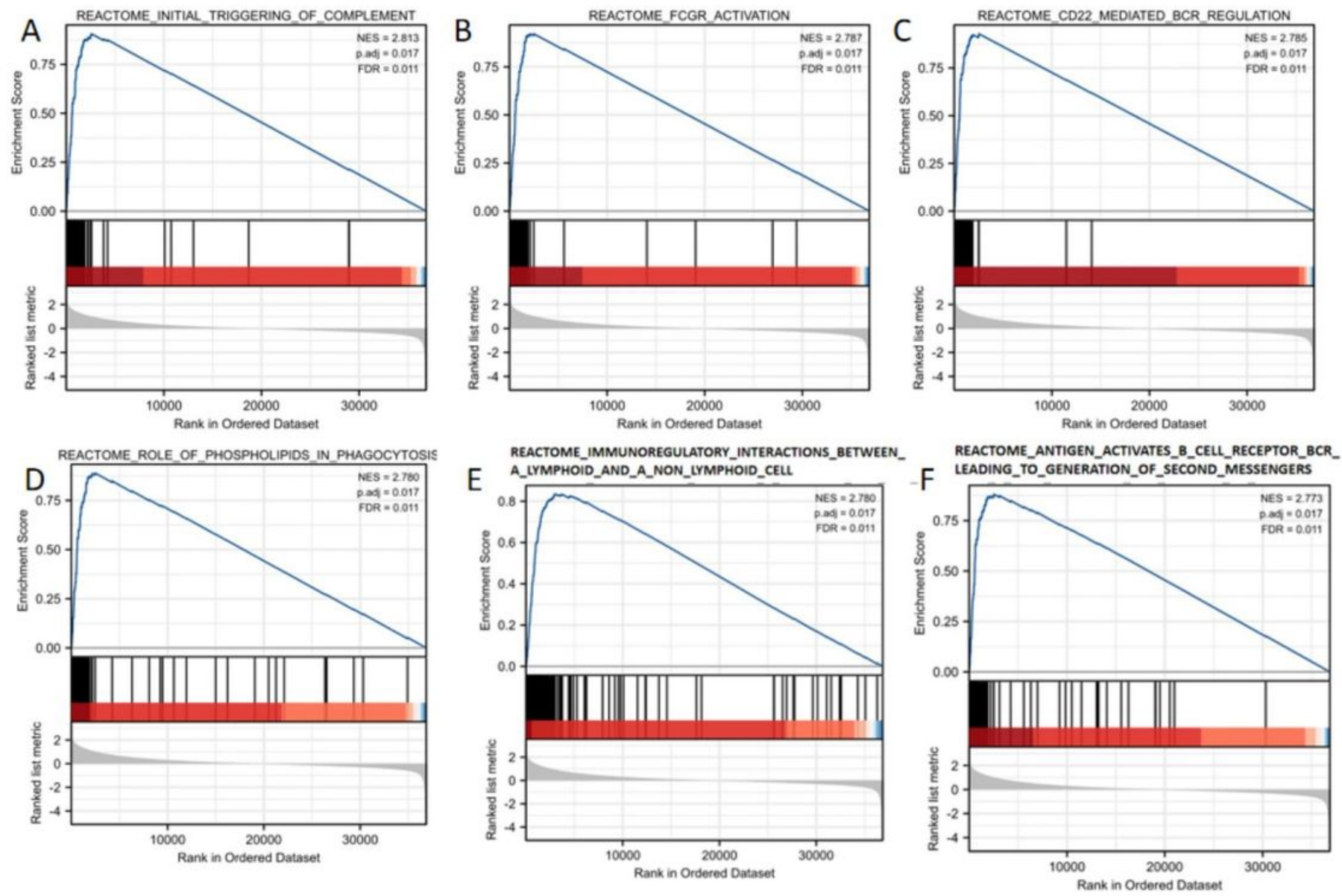

Figure 6

GSEA pathways enriched in gastric carcinoma samples with high NLRP3 expression. The GSEA results showed that "Initial triggering of complement " pathway(A), "FCGR activation " pathway(B),"CD22 mediated BCR regulation” pathway(C),"Role of 
phosholipids in phagocytosis" pathway(D),"Immunoregulatory interactions between a Lymphoid and a non-Lymphoid cell" pathway $(E)$, and "Antigen activates B Cell Receptor (BCR) leading to generation of second messengers" pathway $(F)$ were enriched in samples with high expression of NLRP3. NES, normalized enrichment score.

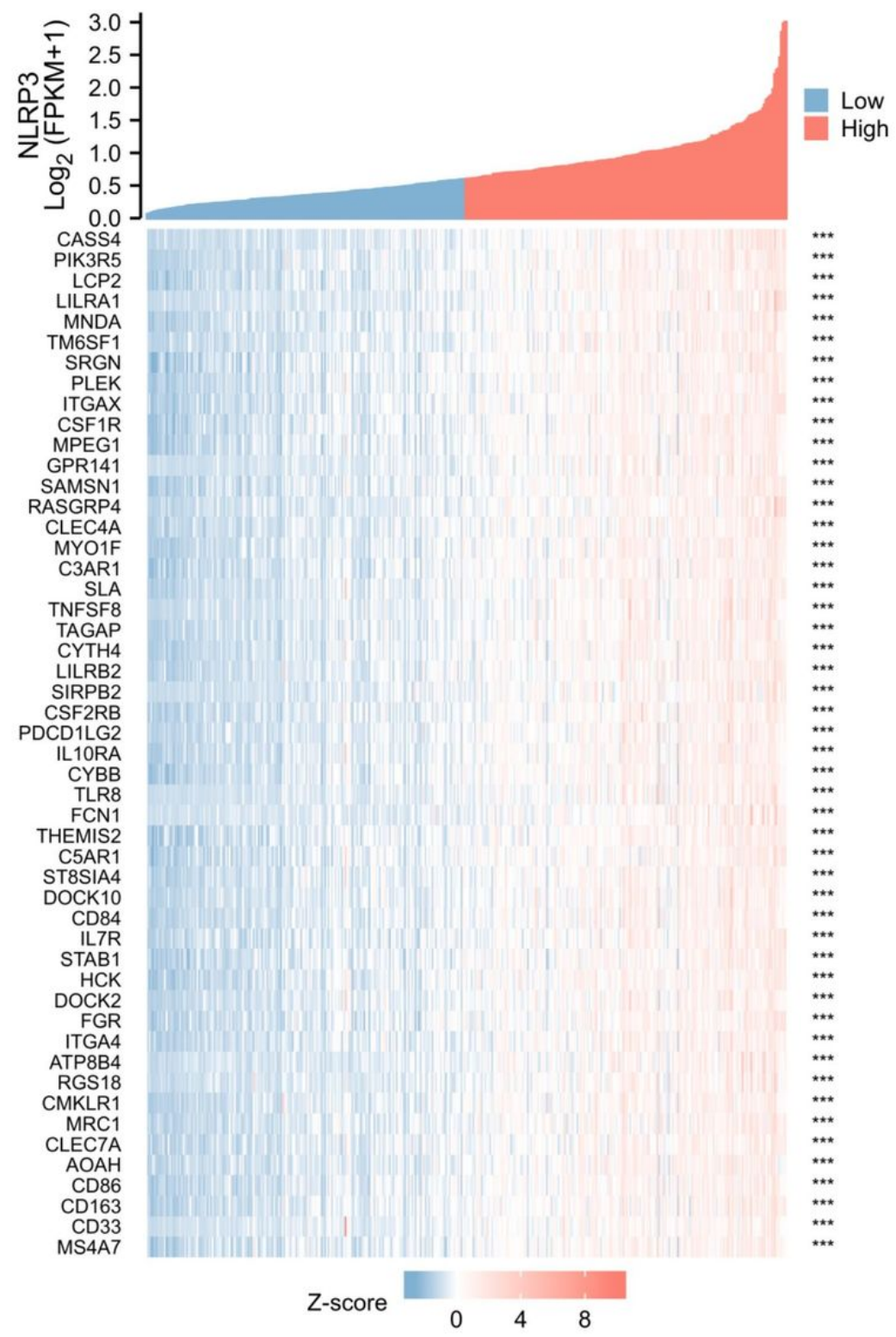

Figure 7

Heatmaps showing the coexpressed genes with NLRP3 in STAD. 

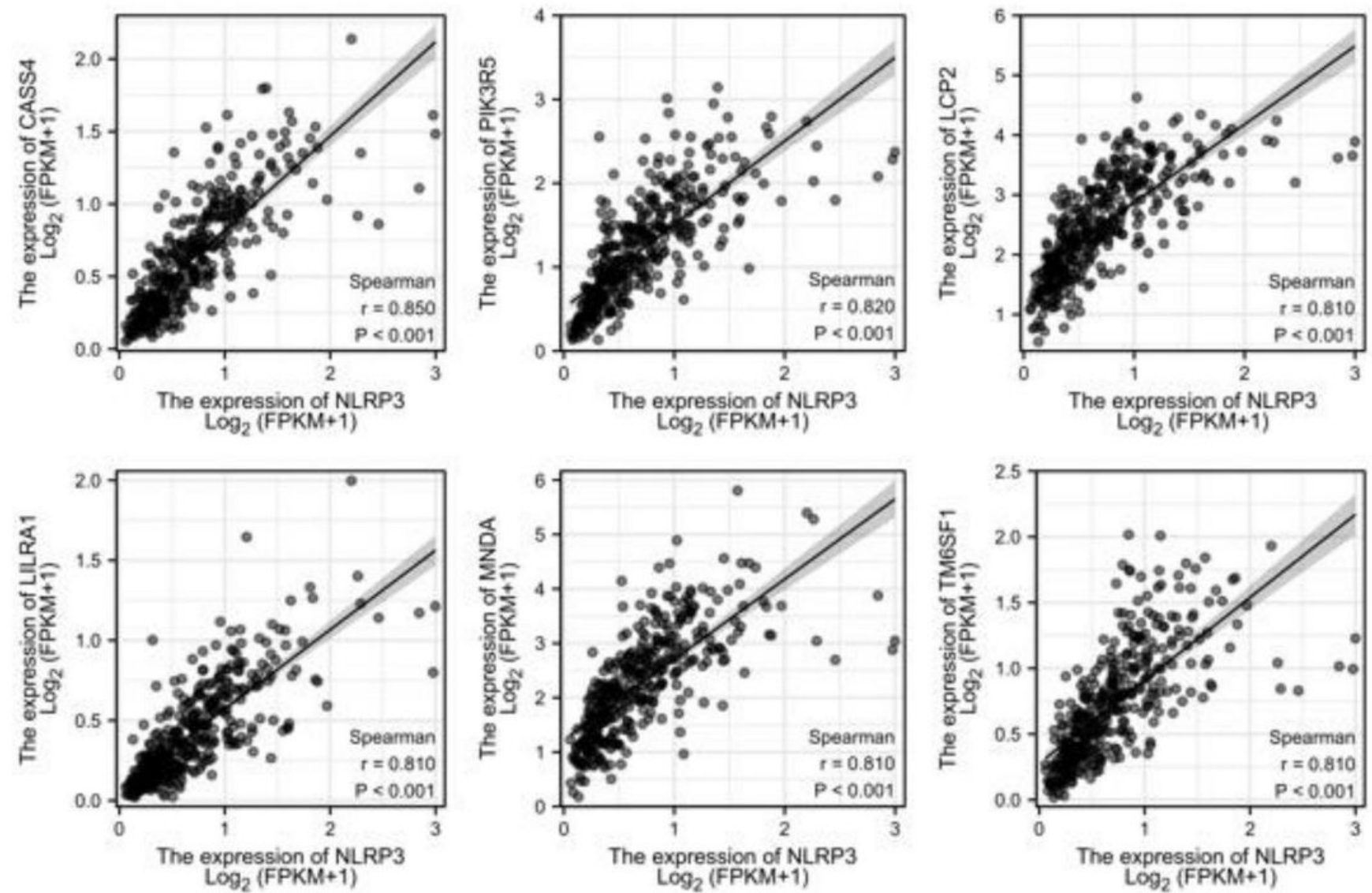

Figure 8

Scatter plots demonstrating the relationship between NLRP3 and CASS4(A), PIK3R5(B), LCP2(C), LILRA1(D),MNDA(E),TM6SF1(F). 
A. LILRA1 (207872_s_at)

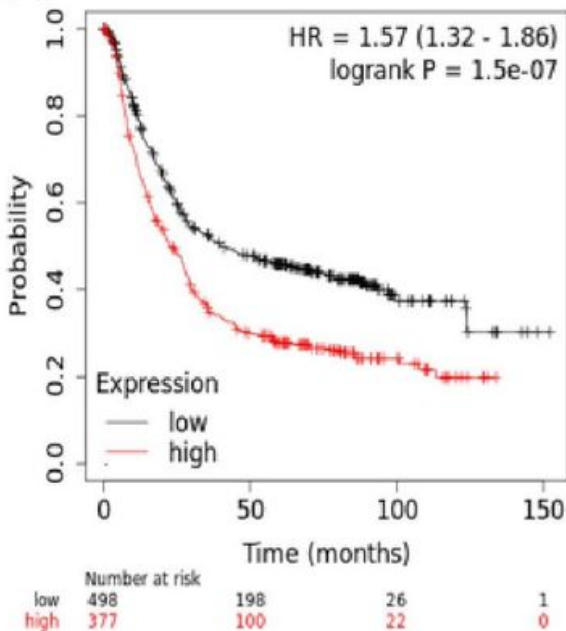

D.

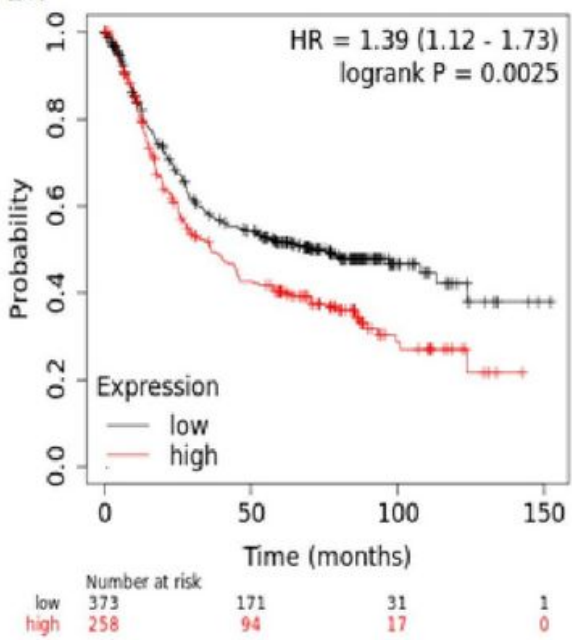

B.

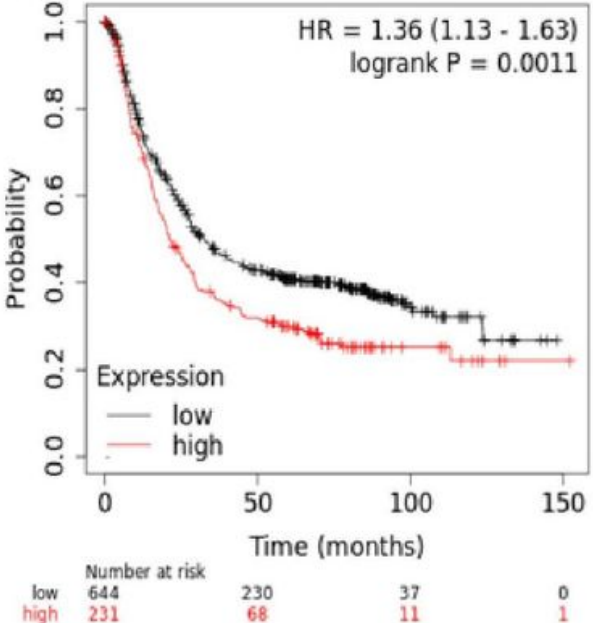

E.

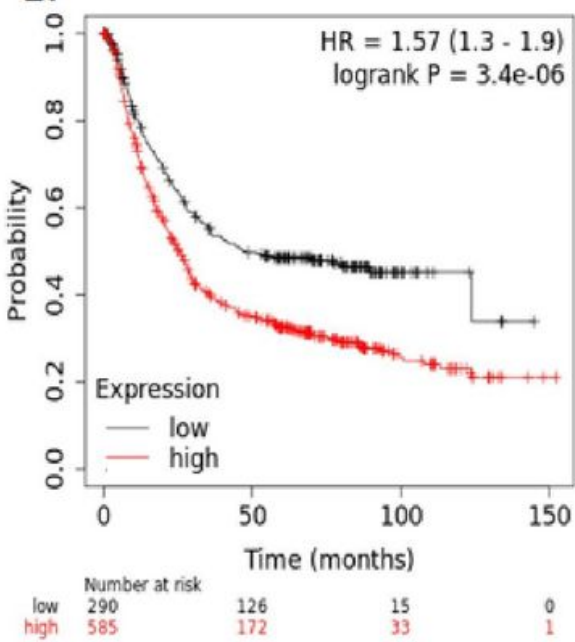

C.

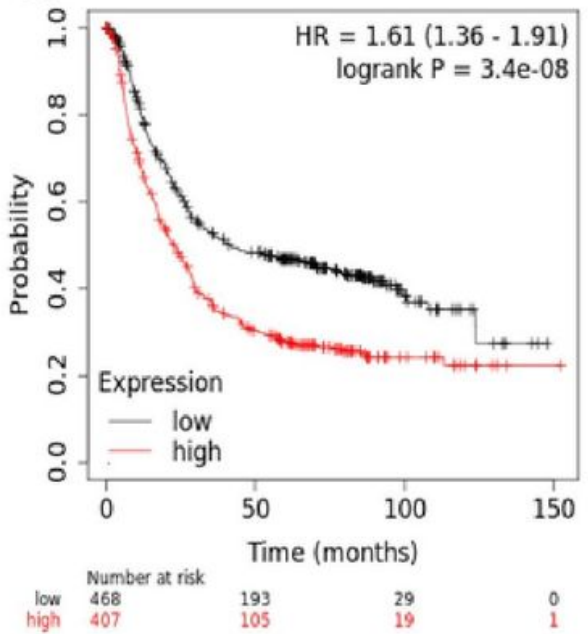

F.

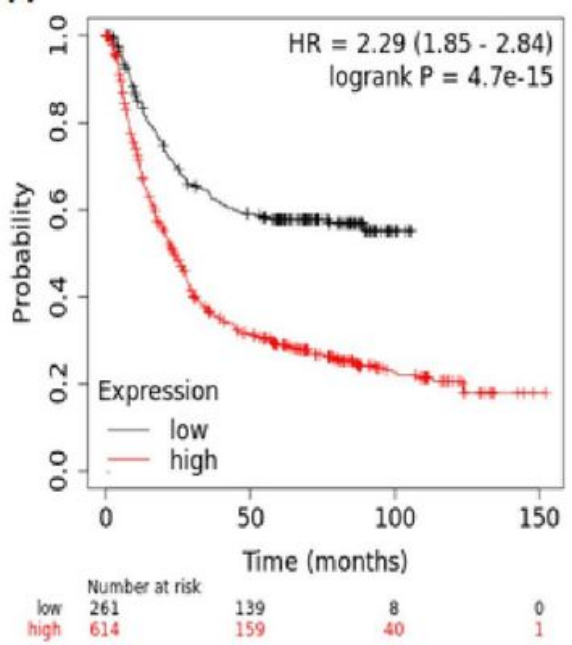

Figure 9

Kaplan-Meier survival analysis of the prognostic significance of genes coexpressed with NLRP3 in STAD. High expression of LILRA1(A), ITGAX(B), PIK3R5(C), RASGRP4(D), MYOIF(E), CSF1R (F) was correlated with worse OS in gastric cancer patients.

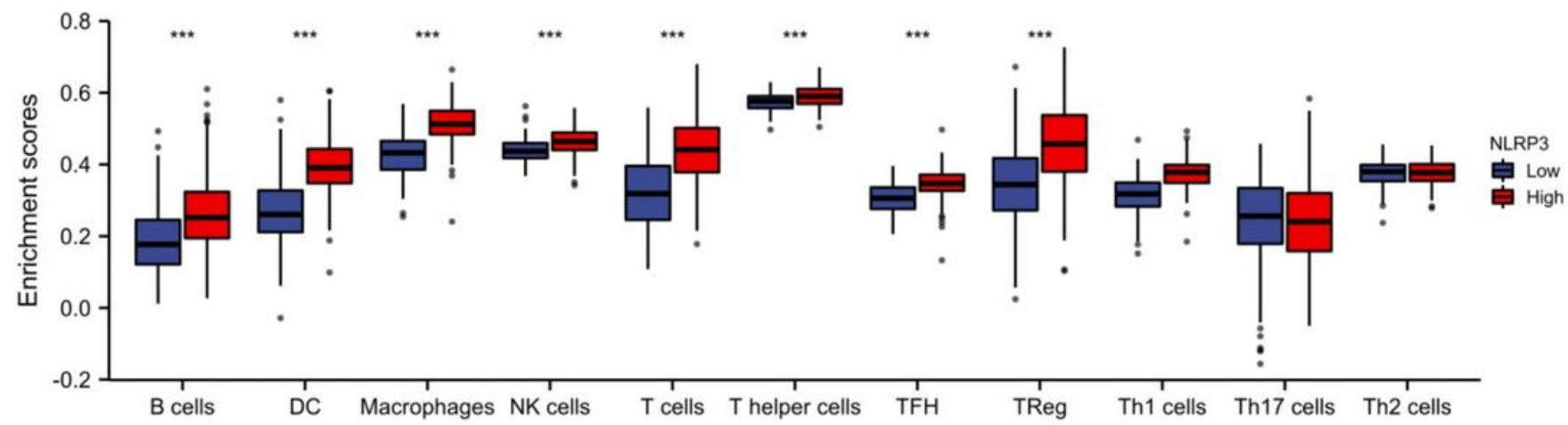

\section{Figure 10}

Correlation analysis of NLRP3 expression and infiltration levels of immune cells in STAD.

$\star \star \star, ~ p<0.001$ 
A.
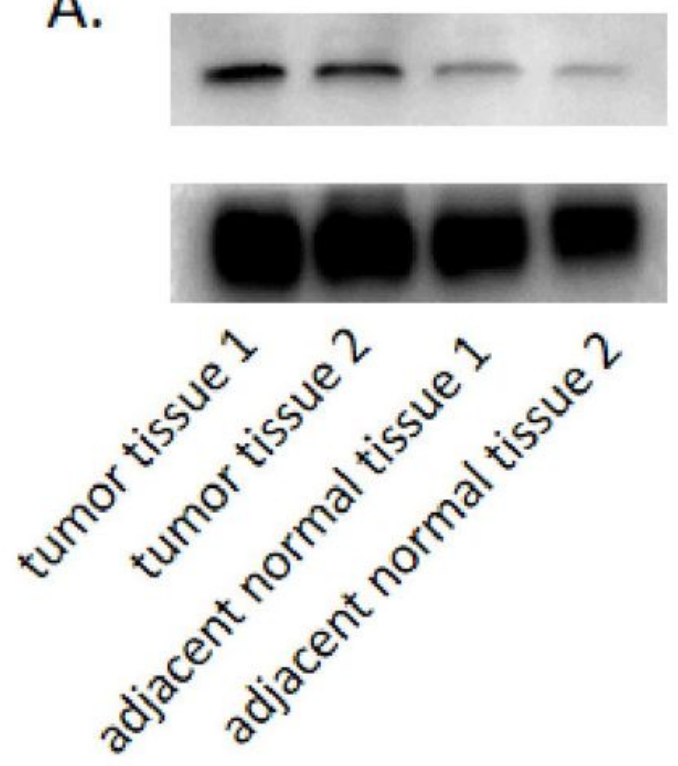

NLRP3

\section{GAPDH}

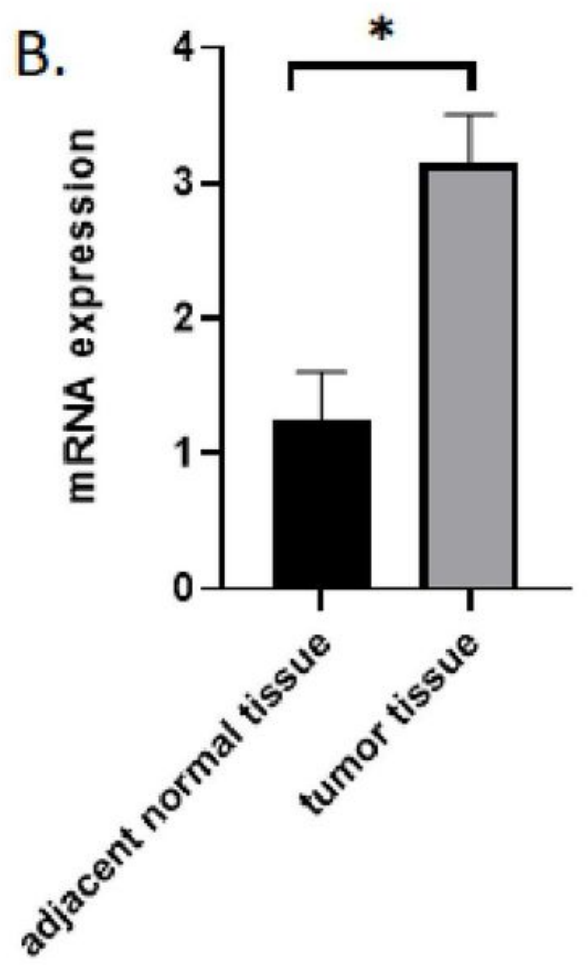

Figure 11

NLRP3 expression in 2 pairs of tumor tissue and adjacent normal tissue.

(A).Western blot verification of NLRP3 expression in tumor tissues and normal tissues.(B).RT-PCR analysis of NLRP3 mRNA expression level .
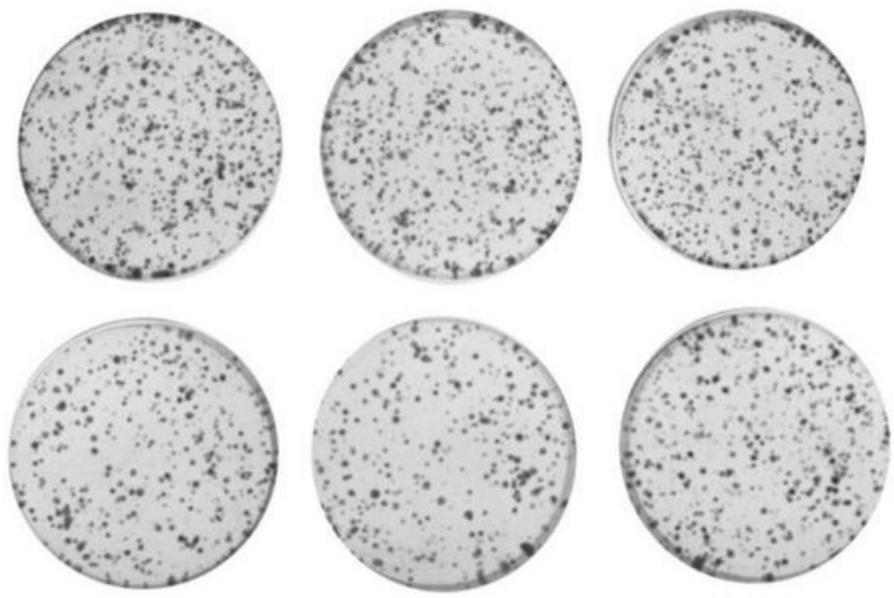

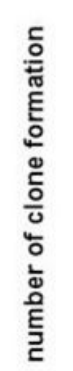

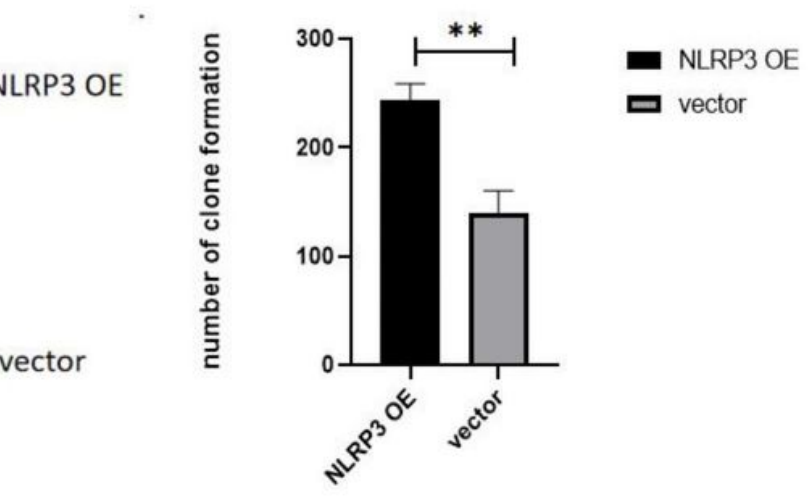

- NLRP3 OE

ㅁ vector

vector

\section{Figure 12}

Colony formation assay in NLRP3-overexpressed SGC-7901 cell and vector transfected cell. Images of clonogenic assay and the bar plot show that NLRP3 overexpressed cells form more colonies than the vector transfected cells. **, $\mathrm{P}<0.01$. 